\title{
CASE REPQORT
}

\section{Bortezomib-Induced Sweet's Syndrome Confirmed by Rechallenge}

\author{
Chrystia M. Zobniw, ${ }^{1}$ Samira A. Saad, ${ }^{2}$ Diana Kostoff, ${ }^{3 *}$ and Bernd G. Barthel ${ }^{4}$ \\ ${ }^{1}$ Department of Pharmacy Services, Roswell Park Cancer Institute, Buffalo, New York; ${ }^{2}$ Department of Pharmacy \\ Services, C.S. Mott Children's Hospital, University of Michigan Health System, Ann Arbor, Michigan; \\ ${ }^{3}$ Department of Pharmacy Services, Henry Ford Health System, Detroit, Michigan; ${ }^{4}$ Department of Hematology \\ and Oncology, Henry Ford Health System, Detroit, Michigan
}

Sweet's syndrome, also known as acute febrile neutrophilic dermatosis, is characterized predominantly by fever, elevated neutrophil count, and erythematous skin lesions composed of plaques and nodules that appear on upper extremities, face, or neck. The incidence of Sweet's syndrome in the general population is unknown due to the rarity of the condition and potential lack of reporting. Bortezomib, an antineoplastic agent that is the standard of care in patients with multiple myeloma, has been reported to be associated with Sweet's syndrome. We describe a 69-year-old man who developed Sweet's syndrome during his initial course (after cycle 4) of bortezomib for treatment of multiple myeloma; he again experienced Sweet's syndrome 3.5 years later when rechallenged with bortezomib (after cycle 5) for treatment of relapsed multiple myeloma. The patient's signs, symptoms, and biopsy results were identical during both presentations of Sweet's syndrome. In both instances, the syndrome spontaneously resolved without incident and without supportive treatment with corticosteroids or antihistamines. To our knowledge, this is the first case report of a patient who developed Sweet's syndrome during an initial course of treatment with bortezomib and after rechallenge with bortezomib for relapsed disease. As proteasome inhibitors continue to be a mainstay of therapy for both treatment and salvage therapy for multiple myeloma, this case demonstrates that rechallenge with bortezomib is an option for patients who develop Sweet's syndrome.

KeY Words Sweet's syndrome, bortezomib, rechallenge, drug induced, adverse reaction multiple myeloma.

(Pharmacotherapy 2014;34(4):e18-e21) doi: 10.1002/phar.1383

Sweet's syndrome, also known as acute febrile neutrophilic dermatosis, is characterized predominantly by fever, elevated neutrophil count, and erythematous skin lesions composed of plaques and nodules that appear on upper extremities, face, or neck. Histologic features include dense mature neutrophil infiltration widely distributed in the upper dermis. ${ }^{1,2}$ The incidence of Sweet's

\footnotetext{
*Address for Correspondence: Diana Kostoff, Clinical Pharmacy Specialist, Hematology/Oncology, Department of Pharmacy, Henry Ford Hospital, 2799 West Grand Boulevard, Detroit, MI 48202- 2689; e-mail: dkostof1@hfhs.org. (C) 2013 American College of Clinical Pharmacy.
}

syndrome in the general population is unknown due to the rarity of the condition; most cases are documented in specific case reports. ${ }^{3}$

Three types of Sweet's syndrome have been distinguished: classic Sweet's syndrome, malignancy-associated Sweet's syndrome, and druginduced Sweet's syndrome., 1 Classic Sweet's syndrome commonly presents in women aged 30-50 years. It may be associated with infection, inflammatory bowel disease, or pregnancy. Malignancy-associated Sweet's syndrome is temporally associated with either an initial diagnosis of malignancy or a leading sign of cancer recurrence. It has been reported with all types of 
cancers; however, 85\% of reported cases had underlying hemopoietic neoplasia. ${ }^{1,2}$ Druginduced Sweet's syndrome is frequently seen in patients treated with granulocyte colony-stimulating factor. A variety of other drugs has also been associated with drug-induced Sweet's syndrome such as antibiotics, oral contraceptives, immunomodulating drugs, and antineoplastic agents. ${ }^{1,2}$

Bortezomib is an antineoplastic agent that has been reported to be associated with Sweet's syndrome. ${ }^{2,4,5}$ It is a selective proteasome inhibitor that is highly effective for the treatment of multiple myeloma and is a reversible inhibitor of the $26 \mathrm{~S}$ proteasome, which is responsible for protein expression leading to the regulation of cellular functions. Its role in multiple myeloma correlates with the inhibition of nuclear factor $(\mathrm{NF})-\kappa \mathrm{B} .^{3}$

To our knowledge, there have been no published case reports documenting the results of rechallenging patients who experienced Sweet's syndrome with bortezomib; we describe a case that occurred in a patient with relapsed multiple myeloma.

\section{Case Report}

A 69-year-old, Caucasian man with a medical history significant for hypertension, asthma, hemochromatosis, and monoclonal gammopathy was diagnosed with smoldering (asymptomatic) multiple myeloma. A bone marrow biopsy confirmed the differential diagnosis. Marrow plasma cells represented $17 \%$ of nucleated cells, and iron was almost absent in stores and erythroblasts. Hemochromatosis was treated with phlebotomies every 3 months and darbepoetin alfa. Myeloma progression was monitored yearly, with no significant changes noted.

A bone marrow biopsy performed approximately 6.5 years after the initial diagnosis of multiple myeloma revealed plasma cells representing $27 \%$ of nucleated cells, indicative of persistent multiple myeloma. Iron stores were found to be absent, similar to the previous biopsy. Cytogenetics were performed, showing a $\mathrm{t}(11: 14)$ translocation, presence of $\mathrm{p} 53$, and $17 \mathrm{p}$ deletion. At this time, the patient's immunoglobulin $\mathrm{G}(\operatorname{Ig} G) \kappa$ monoclonal protein concentration was $2.4 \mathrm{~g} / \mathrm{dl}(>3 \mathrm{~g} / \mathrm{dl}$ at diagnosis is consistent with multiple myleoma). He was started on systemic therapy with lenalidomide and low-dose dexamethasone.

Five months later, the patient's IgG $\mathrm{\kappa}$ monoclonal protein level decreased to $0.7 \mathrm{~g} / \mathrm{dl}$. The patient was evaluated for autologous stem cell transplantation and was determined to be a candidate. Antineoplastic therapy with bortezomib was started 1 month later to prepare for transplantation. The regimen consisted of an intravenous bolus of bortezomib $1.3 \mathrm{mg} / \mathrm{m}^{2}$ on days 1 , 4,8 , and 11 , repeated in cycles of every 21 days. The patient tolerated bortezomib well through cycles 1-3, with no difficulties noted. Two months later, after his final bortezomib treatment during cycle 4 , the patient was noted to have developed erythematous papules over his body and was referred to the dermatology service.

The patient's erythematous papules were mostly scattered on his chest and back, whereas his buttocks contained violaceous patches and papules extending onto the proximal posterior thighs. A 4-mm punch biopsy taken from the left buttock revealed perivascular neutrophilic inflammation and leukocytoclastic vasculitis. The differential diagnosis revealed neutrophilic dermatotoses, specifically Sweet's syndrome. No specific treatment was given, and his papules spontaneously resolved within 10 days. Due to the self-resolution with lack of treatment, his case supported a mild neutrophilic drug reaction to bortezomib.

Three and a half years after the patient's initial treatment with bortezomib, a similar clinical presentation occurred after completion of cycle 5 of bortezomib. He had experienced relapse of multiple myeloma 5 months earlier, during which time his $\operatorname{Ig} G \kappa$ monoclonal protein level steadily rose from $0.2 \mathrm{~g} / \mathrm{dl}$ after autologous stem cell transplantation to $1.2 \mathrm{~g} / \mathrm{dl}$. A subsequent bone marrow biopsy showed plasma cells representing $21 \%$ of nucleated cells, indicating persistent multiple myeloma. A decision was made to undergo a second autologous stem cell transplantation.

Bortezomib therapy was started within 1 month of the patient's relapse of multiple myeloma. The patient received two cycles of an intravenous bolus of bortezomib $1.3 \mathrm{mg} / \mathrm{m}^{2}$ on days $1,4,8$, and 11 , repeated every 21 days. After the second cycle, he started experiencing uncomfortable tingling and numbness in his feet; however, he continued with his treatment. The regimen was changed to subcutaneous administration, with the expectation of decreased neuropathy. Subsequently, the patient developed ecchymotic, erythemic areas surrounding the injection sites, and he was switched to intravenous bortezomib due to lack of improvement of 


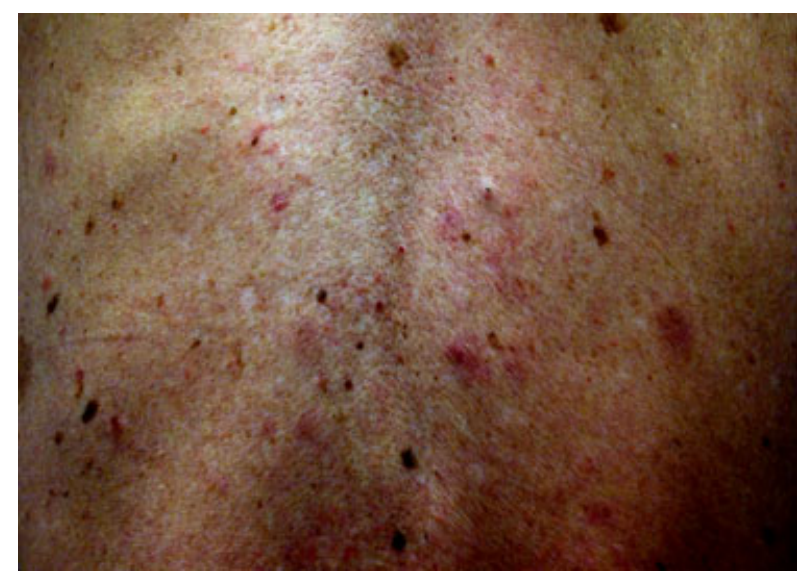

Figure 1. Erythematous papules noted on the patient's upper back after rechallenge with bortezomib.

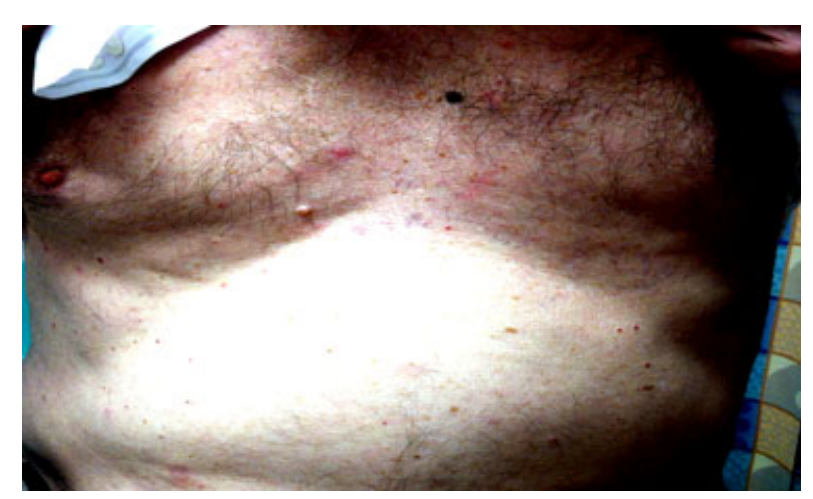

Figure 2. Erythematous papules on the patient's chest and abdomen after rechallenge with bortezomib.

his neuropathy. He continued with this intravenous bortezomib regimen until cycle 5 , at which point he was discovered to have a slightly pruritic eruption on his lower back, with numerous spots on his upper back (Figure 1) and chest and abdomen (Figure 2).

The dermatology service was consulted, and a skin biopsy was conducted that revealed neutrophilic dermatitis with perivascular lymphocytic inflammation, similar to his previous clinical presentation. The biopsy summary concluded that histologic features were consistent with Sweet's syndrome. The dermatology service determined that it would be safe to continue bortezomib treatments unless the rash disseminated further.

\section{Discussion}

Bortezomib is a selective reversible proteasome inhibitor that is highly effective for the treatment of patients with multiple myeloma. Its main role is to target $\mathrm{NF}-\mathrm{\kappa} \mathrm{B}$, a transcription factor that promotes cell proliferation, induces antiapoptotic cell pathways, and regulates the expression of cell surface adhesion molecules involved with inflammation. Bortezomib inhibits $N F-\kappa \quad B$, hence preventing myeloma cells from binding to bone marrow stromal cells and inhibiting angiogenesis. ${ }^{4,5}$ Common adverse effects reported with bortezomib include fatigue, nausea, constipation, peripheral neuropathy, peripheral edema, thrombocytopenia, and neutropenia. ${ }^{6}$ A maculopapular rash was reported in $10-20 \%$ of patients treated for multiple myeloma; however, it has not been well characterized. ${ }^{6}$

Previous reported cases have determined that dermatosis-related symptoms, including rash, are not a contraindication for use. ${ }^{7-10}$ Rash is usually resolved with a combination of topical or systemic corticosteroids and antihistamines. High-potency topical corticosteroids, such as $0.05 \%$ clobetasol propionate, can be applied to the lesions. Systemic corticosteroid therapy is considered the gold standard management approach. Dosing strategies have varied, depending on severity of symptoms, ranging from $1 \mathrm{mg} / \mathrm{kg} /$ day of prednisone to a standard dose of 30-60 mg given as a single oral morning dose. ${ }^{1,2}$ Treatment response to systemic corticosteroids is generally quick, with resolution occurring within hours of exposure.

Prevention of rash with rechallenge is typically accomplished either by prophylactic oral prednisone administration or concurrent intravenous corticosteroids, allowing for continuation of bortezomib therapy. ${ }^{7-10}$ If bortezomib is to be administered subcutaneously, the package insert states that development of local injection site reactions can prompt reconstitution to a lower concentration of $1 \mathrm{mg} / \mathrm{ml}$, versus the conventional concentration of $2.5 \mathrm{mg} / \mathrm{ml}^{6}$

Our patient was administered a bortezomib regimen on two separate occasions separated by approximately 3.5 years and developed identical symptoms and pathologic findings of Sweet's syndrome within a similar time frame-after four cycles of multiple myeloma therapy. The use of single-agent bortezomib, versus a bortezomib and dexamethasone combination, highlights that the patient's increase in neutrophil count was specific to bortezomib alone. Furthermore, use of the Naranjo adverse reaction probability scale indicated a definite relationship (score of 10) between the patient's development of Sweet's syndrome and bortezomib therapy (Appendix 1). 


\section{Conclusion}

To the best of our knowledge, this is the first case report to document a patient who was successfully rechallenged with bortezomib without premedication with corticosteroids after previously developing Sweet's syndrome. However, the exclusivity of this case report lies in the recurrence of Sweet's syndrome after rechallenge of bortezomib. Our patient experienced significant neutrophilic dermatitis with perivascular lymphocytic inflammation after two separate instances of intravenous bortezomib administration separated by 3.5 years, with the first occurring after cycle 4 and the second after cycle 5 . Proteasome inhibitors continue to be a mainstay of therapy for both treatment and salvage therapy for multiple myeloma. Thus, this case report provides insight that rechallenge with bortezomib is an option for patients who develop Sweet's syndrome.

\section{References}

1. Paydas S. Sweet's syndrome: a revisit for hematologists and oncologists. Crit Rev Oncol Hematol 2013;86:85-95.

2. Cohen PR. Sweet's syndrome-a comprehensive review of an acute febrile neutrophilic dermatosis. Orphanet J Rare Dis 2007;2:34.

3. Ginarte M, Toribio J. Sweet syndrome. In: Huag FP, ed. Autoimmune disorders - current concepts and advances from bedside to mechanistic insights. Croatia: InTech, 2011:119-32.

4. Richardson PG, Barlogie B, Berenson J, et al. A phase 2 study of bortezomib in relapsed, refractory myeloma. N Engl J Med 2003;348:2609-17.

5. Roccaro AM, Hideshima T, Raje N, et al. Bortezomib mediates antiangiogenesis in multiple myeloma via direct and indirect effects on endothelial cells. Cancer Res 2006;66:184-91.

6. Millennium Pharmaceuticals. Velcade (Bortezomib) package insert. Cambridge, MA: Millennium Pharmaceuticals; 2012.

7. Villarrubia B, Betlloch I, Mataix J, Lucas A, Botella C. Bortezomib associated rash: a new recognizable and avoidable side-effect. Br J Dermatol 2007;156:784-5.

8. Garcia-Navarro X, Puig L, Fernández-Figueras MT, Dalmau J, Roe E, Alomar A. Bortezomib-associated cutaneous vasculitis. Br J Dermatol 2007;157:799-801.

9. Paiva CM, Kurtis B, Mekki M, Newman MA, Singhal S, Lacouture ME. Neutrophilic dermatitis associated with bortezomib in a patient with multiple myeloma. Ann Oncol 2007;18:1744-5.

10. Murase JE, Wu JJ, Theate I, Cole GW, Barr RJ, Dyson SW. Bortezomib-induced histiocytoid Sweet syndrome. J Am Acad Dermatol 2009;60:496-7.

11. Naranjo CA, Busto U, Sellers EM. A method for estimating the probability of adverse drug reactions. Clin Pharmacol Ther 1981;30:239-45.
Appendix 1. The Patient's Score Determined by Using the Naranjo Adverse Drug Reaction Algorithm.

Naranjo Adverse Drug Reaction Algorithm

\begin{tabular}{cll}
\hline Question & Answer & Points \\
\hline 1. Are there previous & Yes & +1
\end{tabular}

conclusive reports on this

reaction?

2. Did the adverse event

appear after the suspected

drug was given?

3. Did the adverse reaction

Yes

$+2$

improve when the drug was

discontinued or a specific

antagonist was given?

4. Did the adverse reaction

Yes

$+2$

appear when the drug was re-administered?

5. Are there alternative

No

$+2$

causes that could have caused the reaction?

6. Did the reaction reappear when a placebo was given?

7. Was the drug detected in any body fluid in toxic concentrations?

8. Was the reaction more severe when the dose was increased or less severe when the dose was decreased?

9. Did the patient have a

Do not know or not done Do not know or not done

Do not know 0 or not done similar reaction to the same or similar drugs in any previous exposure?

10. Was the adverse event Yes confirmed by any objective evidence?

Total points indicating
(ADR)

Naranjo Scoring key: $>9=$ definite ADR; 5-8 = probable ADR; $1-4=$ possible ADR; $0=$ doubtful ADR. 